\title{
Fatty acid signatures in plasma of captive herring gulls as indicators of demersal or pelagic fish diet
}

\author{
Reijo Käkelä1,*, Anne Käkelä1 ${ }^{1}$, Silke Kahle ${ }^{2}$, Peter H. Becker ${ }^{2}$, Andrew Kelly ${ }^{3}$, \\ Robert W. Furness ${ }^{3}$ \\ ${ }^{1}$ Department of Biology, University of Joensuu, PO Box 111, 80101 Joensuu, Finland \\ ${ }^{2}$ Institute of Avian Research 'Vogelwarte Helgoland', An der Vogelwarte 21, 26386 Wilhelmshaven, Germany \\ ${ }^{3}$ Institute of Biomedical and Life Sciences, Graham Kerr Building, University of Glasgow, Glasgow G12 8QQ, UK
}

\begin{abstract}
Current fishing practices provide scavenging seabirds with discarded demersal fish. In order to study the impact of fishery management measures and alterations in the availability of discards on seabird populations, accurate information on the birds' diet is essential. Studies of pellets and prey remains provide a biased picture and tend to be limited to seabird breeding seasons. Studying biochemical markers in bird tissues can complement other methods. In this study, captive herring gulls Larus argentatus were fed alternating diets of North Atlantic demersal and pelagic fish, and changes in the fatty acid signatures (FAS) of the birds' plasma were followed. The large differences in FAS of demersal and pelagic fish caused several clearly detectable differences in the plasma FAS of the gulls fed on these fish. A change from demersal to pelagic fish or vice versa could be detected in plasma FAS within $5 \mathrm{~d}$ of switching the diet, and transient changes in the gull's nutritional status did not disturb the use of the FAS. Relatively high levels of branched-chain 17:0, 18:1n-7 and 20:4n-6 are indicative of a diet of demersal fish, and high levels of 14:0, 22:1n-11, 20:1n-9, 18:2n-6, 18:3n-3 and 18:4n-3 occur when gulls eat pelagic fish. These reference FAS can be used to estimate the importance of demersal and pelagic fish in the recent diet of scavenging seabirds.
\end{abstract}

KEY WORDS: Fatty acid signatures · Calibration study · Fishery discards · Foraging ecology • Seabirds $\cdot$ Herring gull $\cdot$ Larus argentatus

\section{INTRODUCTION}

Globally, some 25 million t of fish are discarded at sea each year (Alverson et al. 1994) and most of those discards are demersal fish that would not normally be available to seabirds as natural food (Furness 2003). Populations of scavenging seabird species in the North Sea have been estimated to have increased at least 10fold from 1900 to 2000 (Mitchell et al. 2004). One of the reasons for this increase has been the current practices of demersal fisheries that have provided the scavenging seabirds with large amounts of discards, such as undersized haddock Melanogrammus aeglefinus and whiting Merlangius merlangus, and offal (Garthe et al. 1996, Stratoudakis et al. 1998). However, the recent quantities of discards in the North Sea have been relatively low, and are expected to decline even further, because of reduced fish stocks, management measures and more selective fishing gears (ICES 2002). The FAO code of conduct for responsible fisheries also advocates the drastic reduction in the amounts of discarding (FAO 1995). The reduced availability of discards is likely to have consequences for seabird populations, including scavenging species that are of international conservation importance, e.g. the great skua Stercorarius skua in the North Sea (Ratcliffe et al. 1998, 2002), Audouin's gull Larus audouinii and Balearic shearwater Puffinus mauretanicus in the western Mediterranean (Oro \& Ruiz 1997, Oro \& Pradel 2000, Oro et al. 2003). Besides the effects on body condition (Hüppop \& Wurm 2000), the shortage of discards leads to preyswitching by scavenging seabirds such as skuas, including increased predation on smaller seabird species (Oro \& Furness 2002, Votier et al. 2004). 
In order to study the impact of fisheries' discarding rates on seabird populations, it is essential to obtain accurate information on the diet of scavenging seabirds. However, in many cases, the information provided by the conventional studies of pellets or prey remains is very limited, and concerns only the last meal. In addition, these pellets and prey remains do not represent assimilated food, and the prey item data are biased towards prey types that have low digestibility and leave clear remains (Frank 1992, Hobson et al. 1994, Votier et al. 2001, 2003). Due to the limitations of the traditional methods, and because direct observation of feeding is rarely possible, there is a growing interest in the use of biochemical markers in tissues of top predators as indicators of diet.

Fatty acids and stable isotopes of nitrogen and carbon are candidate biochemical markers to assess relative contributions of different dietary items in the diet of carnivorous mammals, birds and fish (Rau et al. 1992, Hilderbrand et al. 1996, Iverson et al. 1997 , Bearhop et al. 2002, Bradshaw et al. 2003). Stable isotope ratios are normally used to indicate the trophic level or large-scale differences in the diets of predators. In contrast, fatty acid analysis of predator tissues may have the potential to reveal more detailed information on the predator's diet (e.g. Iverson et al. 2004). We tested the applicability of both fatty acids and stable isotopes to indicate demersal and pelagic fish diet by feeding trials using captive herring gulls Larus argentatus as model scavenging seabirds. The results of the stable isotope approach will be reported elsewhere (A. Kelly et al. unpubl.). This study focuses on the use of gas-liquid chromatographic data of plasma fatty acid composition as an indication of prey type.

Since the advent of modern gas-liquid chromatography and high-resolution capillary columns, reports on detailed fatty acid compositions in various animal tissues have accumulated showing that the fatty acid composition of predator tissues are affected by dietary fatty acids (Rouvinen \& Kiiskinen 1989, Käkelä \& Hyvärinen 1996, Käkelä et al. 2001, Dahl et al. 2003, Iverson et al. 2004). The fatty acid composition of storage so-called neutral lipids is normally closer to the ingested mixture of fatty acids than the composition of structural lipids. However, during breeding season, and in the case of protected bird populations sampling of the blood, which contains both structural and neutral lipids, is safer for the birds than taking adipose tissue biopsy.

At present, the chromatographic analyses of detailed fatty acid compositions, i.e. fatty acid signatures (FAS), of the predator and prey are usually combined with the use of either multivariate statistical analyses (mainly principal component analysis, PCA) or classification and regression trees (CART) (e.g. Iverson et al. 1997,
Olsen \& Grahl-Nielsen 2003). In addition, a statistical model for prey identification in particular was published recently (Iverson et al. 2004). However, distinguishing between different dietary groups by means of these advanced analyses of FAS necessitates that the prey species have distinct differences in their FAS. Another prerequisite is that these differences are transferred into the tissues of the predators efficiently enough to produce significant compositional changes that can be linked with the prey type. It should be noted that fatty acids are extensively metabolised in the predator's tissues, and that the efficiency of accumulation and degree of modification depends on the type of tissue (Brockerhoff et al. 1967, Soriguer et al. 2000). Thus, the fatty acid compositions are not transferred intact, and, as shown by Grahl-Nielsen et al. (2003), the FAS may not be powerful enough to reveal all of the predator-prey interactions. In addition, in order to interpret the information from the FAS correctly, it is crucial to know how long it takes for the FAS in a particular tissue to react to a change in the diet. Thus, the use of FAS for assessing the prey of wild seabirds or other top predators requires careful feeding experiments in which representative model species are fed the most common prey items, and the modifications due to the metabolism of the predator are determined.

In this calibration study we studied the transfer efficiencies and turnover rates of fatty acids characteristic of pelagic or demersal fish in the plasma FAS of herring gulls. Our aim was to test the suitability of FAS as a means of discriminating between pelagic and demersal fish in seabird diet, and to create reference data that can be used when assessing the relative contributions of demersal discarded fish and pelagic fish in the diet of scavenging seabirds in the North Sea and other regions of the Atlantic Ocean.

\section{MATERIALS AND METHODS}

Feeding trial and sampling. Ten captive-bred adult herring gulls were housed in outdoor aviaries $(3 \times 6.7 \times$ $2.1 \mathrm{~m})$ at the Institute of Avian Research 'Vogelwarte Helgoland', Wilhelmshaven, Germany on a maintenance diet of dried terrestrial food (main fatty acid composition as mol\%: 14:0 2.4, 16:0 37.5, 18:0 5.3, 18:1n-9 29.8, 18:1n-7 1.1, 18:2n-6 16.3, 18:3n-3 1.2). The birds were divided into 3 groups and were fed diets that comprised different species of North Atlantic fish; the pelagic species herring (Clupea harengus, undersized young individuals, $1.8 \pm 0.6 \% \mathrm{lipid}, \mathrm{wt} / \mathrm{wt}$ ), sardine (Sardina pilchardus, $10.8 \pm 3.0 \%$ lipid) and mackerel (Scomber scombrus, $8.3 \pm 1.2 \%$ lipid), the demersal species haddock $(2.0 \pm 0.7 \%$ lipid $)$, whiting 
$(1.0 \pm 0.4 \%$ lipid $)$ and flatfish sp. (mainly plaice Pleuronectes platessa, $1.0 \pm 0.4 \%$ lipid) over a period of 12 wk (fatty acid compositions in Table 1). The fish were obtained from commercial and research fishing vessels in the southern North Sea and eastern Atlantic sampling small fish typical of the sizes discarded by fisheries. In Group A ( $=4)$ the birds were first given a diet of pelagic sardines and then switched to a different fish species every $2 \mathrm{wk}$ in the order: haddock-herring-flatfish sp.-mackerel-whiting, in a way that diets of pelagic and demersal fish alternated. In Group B (N $=3$ ) the gulls were started on a haddock (demersal) diet, which was then switched to herring (pelagic) after 6 wk. Group $\mathrm{C}(\mathrm{N}=3)$ was started on a sardine (pelagic) diet and after 6 wk continued on flatfish (demersal). The gulls were offered $1.0 \mathrm{~kg}$ of fish daily for every 3 to 4 birds, which was all consumed, except in the case of the sardines, where only $0.5 \mathrm{~kg}$ was eaten per day. The gulls were fed in the morning after the sampling of blood. Thus, the time interval from the last feeding to the sampling was approximately $24 \mathrm{~h}$.

The blood samples were taken under licence of the Bezirksregierung Weser-Ems, Oldenburg. Blood was sampled by venipuncture from the metatarsal vein every $5 \mathrm{~d}$ for Group A birds, and every $7 \mathrm{~d}$ for birds from Groups B and C via a 23 gauge needle into a syringe lined with $100 \mu \mathrm{l}$ of EDTA to prevent clotting. The blood was centrifuged (for 15 min with $8900 \times g$ at $4^{\circ} \mathrm{C}$ ) to separate plasma, which was stored at $-80^{\circ} \mathrm{C}$ for 2 to 3 mo before analysis. The red blood cell fraction was removed and stored at $-80^{\circ} \mathrm{C}$ prior to stable isotope analysis, the results of which will be reported elsewhere.

Fatty acid analysis. Fatty acid composition was determined in the homogenates of the whole fish and herring gull plasma. Subsamples were transmethylated according to the recommendations of Christie (1993) by heating with $1 \% \mathrm{H}_{2} \mathrm{SO}_{4}$ in methanol under nitrogen atmosphere, and the formed fatty acid methyl esters (FAME) extracted with hexane in 2 steps. The methylation method was tested in our laboratory with fish and bird samples and found to produce identical results compared to common alternative methods using methanolic boron trifluoride or hydrochloric acid as reagent. The dried and concentrated FAME were analysed by a gas-liquid chromatograph equipped with 2 injectors and flame ionisation and mass detectors (GC-FID and GC-MS, respectively) (6890N network GC system with autosampler, FID detector and 5973 mass selective detector, Agilent). Both operating lines were equipped with a DB-wax capillary column ( $30 \mathrm{~m}$, ID $0.25 \mathrm{~mm}$, film $0.25 \mu \mathrm{m}, \mathrm{J} \& W$ Scientific). A volume of $2 \mu \mathrm{l}$ was injected using a split ratio of 1:20. The injectors were set at $250^{\circ} \mathrm{C}$, and the FID and mass interphase were at 250 and $200^{\circ} \mathrm{C}$, respectively.
Helium was used as a carrier gas $\left(1.8 \mathrm{ml} \mathrm{min}{ }^{-1}\right.$ for FID line and $1.0 \mathrm{ml} \mathrm{min}^{-1}$ for mass detecting line). The initial oven temperature of $180^{\circ} \mathrm{C}$ was held for $8 \mathrm{~min}$, programmed to rise $3^{\circ} \mathrm{C} \mathrm{min}^{-1}$ to a final temperature of $210^{\circ} \mathrm{C}$, which was held for $25 \mathrm{~min}$. The detected peaks were integrated and the mass spectra extracted using Agilent ChemStation software. The FAME were identified based on retention time, mass spectrum and comparisons with authentic (Sigma) and natural standards of known composition and published reference spectra (Christie W.W., www.lipidlibrary.co.uk/ masspec.html). Quantifications were based on FID responses corrected according to the theoretical response factors (Ackman 1992) and calibrations with quantitative authentic standards. The fatty acid proportions were calculated as mol\%, and the fatty acids were marked by using the abbreviations: [carbon number]:[number of double bonds]n-[position of the first double bond calculated from the methyl end] (e.g. $22: 6 n-3)$. All the quantitatively important polyunsaturated fatty acids (PUFA) were methylene-interrupted.

Lipid analysis. To study the influence of plasma lipid profile on the FAS, the lipid class composition of plasma was analysed using a thin-layer chromatography-FID analyser (TLC-FID, Iatroscan new Mark V, Iatron Laboratories). The plasma total lipids of the Group B gulls were extracted with chloroformmethanol (2:1, vol/vol) according to Folch et al. (1957) and separated on Chromarods using petrol etherdiethyl ether-formic acid (67/2.2/0.75 by vol) as developing solution. Two replicates were analysed and the detector responses were calibrated with authentic quantitative lipid standards (Sigma). The lipid contents of the dietary fish were determined according to the same procedure.

Multivariate analysis of the fatty acid data. To analyse the relationships between FAS of the herring gull plasma samples the data were subjected to multivariate principal component analysis (PCA) using the SIRIUS 6.5 software package (Pattern Recognition Systems) (Kvalheim \& Karstang 1987). The data were standardized, and the relative positions of the samples and variables were plotted using 2 new coordinates, the principal components PC 1 and PC 2, describing the largest and second largest variance among the samples.

\section{RESULTS}

\section{Fatty acid signatures of demersal and pelagic fishes}

The FAS of the total lipids of demersal and pelagic fish species differed in many respects (Table 1). In saturated fatty acids, the demersal species were rich in 18:0 
Table 1. Fatty acid composition (mol\%) in the dietary fish $(\mathrm{N}=6$ in each) and plasma of the herring gulls fed these fish ( $\mathrm{N}=3$ to 4 , only the samples representing the longest feeding time available on each specific diet were included). The letters following the SD-value refer to one-way analysis of variance (ANOVA) followed by the Newman-Keuls test. The means with no common letter differ at $\mathrm{p}<0.05$ level (the smallest mean is marked with A). If the data failed the normality or equal variance tests (some fatty acids in the data of herring gull plasma) the significance after Kruskal-Wallis test is given on the right

\begin{tabular}{|c|c|c|c|c|c|c|c|c|c|c|c|c|}
\hline \multirow[t]{3}{*}{ Fatty acid } & \multicolumn{12}{|c|}{ Dietary fish $(\mathbf{N}=\mathbf{6})$} \\
\hline & \multicolumn{2}{|c|}{ Haddock } & \multicolumn{2}{|c|}{ Whiting } & \multicolumn{2}{|c|}{ Flatfish } & \multicolumn{2}{|c|}{ Herring } & \multicolumn{2}{|c|}{ Sardine } & \multicolumn{2}{|c|}{ Mackerel } \\
\hline & Mean & $\mathrm{SD}$ & Mean & $\mathrm{SD}$ & Mean & $\mathrm{SD}$ & Mean & $\mathrm{SD}$ & Mean & $\mathrm{SD}$ & Mean & $\mathrm{SD}$ \\
\hline $14: 0$ & 3.24 & $0.60 \mathrm{~B}$ & 1.15 & $0.40 \mathrm{~A}$ & 2.91 & $1.03 \mathrm{~B}$ & 5.42 & $1.44 \mathrm{C}$ & 8.90 & $0.89 \mathrm{D}$ & 7.35 & $1.47 \mathrm{D}$ \\
\hline $15: 0$ iso & 0.27 & $0.02 \mathrm{C}$ & 0.15 & $0.02 \mathrm{~A}$ & 0.26 & $0.07 \mathrm{C}$ & 0.20 & $0.04 \mathrm{~B}$ & 0.19 & $0.03 \mathrm{~B}$ & 0.29 & $0.02 \mathrm{C}$ \\
\hline $15: 0$ & 0.81 & $0.11 \mathrm{CD}$ & 0.64 & $0.09 \mathrm{~B}$ & 0.88 & $0.04 \mathrm{D}$ & 0.48 & $0.04 \mathrm{~A}$ & 0.83 & $0.11 \mathrm{CD}$ & 0.70 & $0.14 \mathrm{BC}$ \\
\hline 16:0 iso & 0.20 & $0.08 \mathrm{~B}$ & 0.29 & $0.06 \mathrm{C}$ & 0.38 & $0.07 \mathrm{D}$ & 0.10 & $0.03 \mathrm{~A}$ & 0.08 & $0.02 \mathrm{~A}$ & 0.11 & $0.05 \mathrm{~A}$ \\
\hline $16: 0$ & 15.24 & $0.95 \mathrm{~A}$ & 17.91 & $0.25 \mathrm{~B}$ & 15.81 & $1.10 \mathrm{~A}$ & 20.50 & $2.41 \mathrm{~B}$ & 19.37 & $1.26 \mathrm{~B}$ & 15.87 & $3.32 \mathrm{~A}$ \\
\hline $16: 1 n-9$ & 0.50 & $0.11 \mathrm{C}$ & 0.35 & $0.03 \mathrm{~B}$ & 0.90 & $0.18 \mathrm{D}$ & 0.27 & $0.08 \mathrm{AB}$ & 0.20 & $0.04 \mathrm{~A}$ & 0.23 & $0.04 \mathrm{~A}$ \\
\hline $16: 1 n-7$ & 4.87 & $0.67 \mathrm{~B}$ & 2.98 & $0.99 \mathrm{~A}$ & 6.20 & $1.10 \mathrm{~B}$ & 3.45 & $0.59 \mathrm{~A}$ & 10.17 & $1.49 \mathrm{C}$ & 5.25 & $1.07 \mathrm{~B}$ \\
\hline $16: 1 n-5$ & 0.34 & $0.05 \mathrm{~A}$ & 0.32 & $0.06 \mathrm{~A}$ & 0.31 & $0.04 \mathrm{~A}$ & 0.32 & $0.07 \mathrm{~A}$ & 0.43 & $0.05 \mathrm{~B}$ & 0.32 & $0.06 \mathrm{~A}$ \\
\hline $16: 2 n-4$ & 0.18 & $0.04 \mathrm{~A}$ & 0.19 & $0.04 \mathrm{~A}$ & 0.44 & $0.20 \mathrm{~B}$ & 0.22 & $0.07 \mathrm{~A}$ & 0.93 & $0.13 \mathrm{C}$ & 0.31 & $0.10 \mathrm{~B}$ \\
\hline $16: 3 n-4$ & 0.10 & $0.03 \mathrm{~A}$ & 0.09 & $0.04 \mathrm{~A}$ & 0.26 & $0.14 \mathrm{~B}$ & 0.15 & $0.05 \mathrm{~A}$ & 0.40 & $0.06 \mathrm{C}$ & 0.15 & $0.05 \mathrm{~A}$ \\
\hline $16: 4 n-1$ & 0.09 & $0.05 \mathrm{~A}$ & 0.11 & $0.04 \mathrm{~A}$ & 0.40 & $0.29 \mathrm{~B}$ & 0.15 & $0.08 \mathrm{~A}$ & 0.86 & $0.15 \mathrm{C}$ & 0.36 & $0.15 \mathrm{~B}$ \\
\hline $17: 0$ iso & 0.46 & $0.11 \mathrm{C}$ & 0.71 & $0.09 \mathrm{D}$ & 0.87 & $0.07 \mathrm{E}$ & 0.15 & $0.04 \mathrm{~A}$ & 0.12 & $0.02 \mathrm{~A}$ & 0.27 & $0.09 \mathrm{~B}$ \\
\hline 17:0 anteiso & 0.31 & $0.07 \mathrm{~B}$ & 0.57 & $0.07 \mathrm{C}$ & 0.80 & $0.11 \mathrm{D}$ & 0.13 & $0.02 \mathrm{~A}$ & 0.11 & $0.02 \mathrm{~A}$ & 0.14 & $0.04 \mathrm{~A}$ \\
\hline $17: 0$ & 0.63 & $0.22 \mathrm{~B}$ & 0.88 & $0.15 \mathrm{C}$ & 0.93 & $0.22 \mathrm{C}$ & 0.26 & $0.04 \mathrm{~A}$ & 0.56 & $0.10 \mathrm{~B}$ & 0.47 & $0.20 \mathrm{~B}$ \\
\hline $17: 1 n-8$ & 0.66 & $0.16 \mathrm{C}$ & 0.65 & $0.14 \mathrm{C}$ & 0.84 & $0.12 \mathrm{C}$ & 0.21 & $0.07 \mathrm{~A}$ & 0.24 & $0.01 \mathrm{~A}$ & 0.41 & $0.15 \mathrm{~B}$ \\
\hline $18: 0$ & 3.98 & $0.92 \mathrm{~B}$ & 6.29 & $0.15 \mathrm{D}$ & 5.14 & $1.06 \mathrm{C}$ & 2.50 & $0.58 \mathrm{~A}$ & 2.96 & $0.39 \mathrm{AB}$ & 3.02 & $0.85 \mathrm{AB}$ \\
\hline 18:1n-13 & 0.22 & $0.17 \mathrm{C}$ & 0.90 & $0.32 \mathrm{D}$ & 1.36 & $0.53 \mathrm{D}$ & 0.03 & $0.01 \mathrm{~B}$ & 0.02 & $0.00 \mathrm{~B}$ & 0.01 & $0.01 \mathrm{~A}$ \\
\hline $18: 1 n-9+11$ & 12.86 & $1.41 \mathrm{C}$ & 8.83 & $0.84 \mathrm{~B}$ & 6.46 & $0.69 \mathrm{~A}$ & 9.65 & $1.71 \mathrm{~B}$ & 5.66 & $0.24 \mathrm{~A}$ & 11.43 & $2.97 \mathrm{~B}$ \\
\hline $18: 1 n-7$ & 3.66 & $0.87 \mathrm{~B}$ & 4.84 & $0.20 \mathrm{C}$ & 5.22 & $0.38 \mathrm{D}$ & 2.83 & $0.56 \mathrm{~A}$ & 2.36 & $0.12 \mathrm{~A}$ & 2.33 & $0.92 \mathrm{~A}$ \\
\hline $18: 1 n-5$ & 0.52 & $0.12 \mathrm{D}$ & 0.59 & $0.14 \mathrm{D}$ & 0.33 & $0.04 \mathrm{~B}$ & 0.53 & $0.16 \mathrm{D}$ & 0.15 & $0.01 \mathrm{~A}$ & 0.40 & $0.02 \mathrm{C}$ \\
\hline $18: 2 n-6$ & 1.58 & $0.30 \mathrm{D}$ & 0.49 & $0.07 \mathrm{~A}$ & 0.61 & $0.03 \mathrm{~B}$ & 1.70 & $0.18 \mathrm{D}$ & 1.26 & $0.13 \mathrm{C}$ & 1.55 & $0.26 \mathrm{D}$ \\
\hline $18: 2 n-4$ & 0.13 & $0.03 \mathrm{~B}$ & 0.31 & $0.03 \mathrm{D}$ & 0.40 & $0.09 \mathrm{E}$ & 0.11 & $0.02 \mathrm{~A}$ & 0.18 & $0.02 \mathrm{C}$ & 0.14 & $0.02 \mathrm{~B}$ \\
\hline $18: 3 n-3$ & 1.24 & $0.50 \mathrm{~B}$ & 0.15 & $0.04 \mathrm{~A}$ & 0.21 & $0.07 \mathrm{~A}$ & 0.86 & $0.19 \mathrm{~B}$ & 0.87 & 0.13 B & 1.33 & $0.39 \mathrm{~B}$ \\
\hline $18: 4 n-3$ & 2.42 & $0.99 \mathrm{C}$ & 0.29 & $0.05 \mathrm{~A}$ & 0.47 & $0.20 \mathrm{~A}$ & 1.35 & $0.74 \mathrm{~B}$ & 1.70 & $0.25 \mathrm{~B}$ & 3.78 & $1.28 \mathrm{D}$ \\
\hline 20:1n-11 & 1.25 & $0.53 \mathrm{AB}$ & 0.84 & $0.32 \mathrm{~A}$ & 1.61 & $0.43 \mathrm{~B}$ & 0.72 & $0.23 \mathrm{~A}$ & 0.70 & $0.15 \mathrm{~A}$ & 0.73 & $0.25 \mathrm{~A}$ \\
\hline 20:1n-9 & 2.61 & $0.80 \mathrm{~B}$ & 1.09 & $0.10 \mathrm{~A}$ & 0.90 & $0.25 \mathrm{~A}$ & 5.16 & $2.81 \mathrm{C}$ & 5.07 & $1.34 \mathrm{C}$ & 8.00 & $3.30 \mathrm{C}$ \\
\hline $20: 1 n-7$ & 1.02 & $0.81 \mathrm{~B}$ & 1.00 & $0.18 \mathrm{~B}$ & 1.68 & $0.46 \mathrm{C}$ & 0.18 & $0.03 \mathrm{~A}$ & 0.18 & $0.01 \mathrm{~A}$ & 0.32 & $0.23 \mathrm{~A}$ \\
\hline $20: 2 n-6$ & 0.61 & $0.20 \mathrm{C}$ & 0.35 & $0.07 \mathrm{~B}$ & 0.37 & $0.08 \mathrm{~B}$ & 0.23 & $0.04 \mathrm{~A}$ & 0.21 & $0.03 \mathrm{~A}$ & 0.31 & $0.09 \mathrm{AB}$ \\
\hline $20: 4 n-6$ & 1.71 & $0.57 \mathrm{~B}$ & 3.26 & $0.32 \mathrm{C}$ & 3.31 & $0.23 \mathrm{C}$ & 0.95 & $0.29 \mathrm{~A}$ & 0.66 & $0.14 \mathrm{~A}$ & 0.69 & $0.23 \mathrm{~A}$ \\
\hline $20: 4 n-3$ & 0.72 & $0.04 \mathrm{D}$ & 0.50 & $0.13 \mathrm{~B}$ & 0.45 & $0.03 \mathrm{~A}$ & 0.63 & $0.06 \mathrm{C}$ & 0.72 & $0.06 \mathrm{D}$ & 1.07 & $0.17 \mathrm{E}$ \\
\hline $20: 5 n-3$ & 11.97 & $1.39 \mathrm{C}$ & 13.79 & $0.81 \mathrm{D}$ & 19.29 & $2.81 \mathrm{E}$ & 8.20 & $1.62 \mathrm{~B}$ & 9.48 & $0.89 \mathrm{~B}$ & 5.92 & $0.73 \mathrm{~A}$ \\
\hline $21: 5 n-3$ & 0.25 & $0.03 \mathrm{~B}$ & 0.34 & $0.08 \mathrm{C}$ & 0.55 & $0.06 \mathrm{D}$ & 0.18 & $0.05 \mathrm{~A}$ & 0.30 & $0.02 \mathrm{C}$ & 0.34 & $0.06 \mathrm{C}$ \\
\hline $22: 1 n-11$ & 1.96 & $0.88 \mathrm{~B}$ & 0.14 & $0.07 \mathrm{~A}$ & 0.13 & $0.07 \mathrm{~A}$ & 8.24 & $4.97 \mathrm{C}$ & 7.14 & $2.08 \mathrm{C}$ & 10.32 & $4.88 \mathrm{C}$ \\
\hline $22: 1 n-9$ & 0.31 & $0.12 \mathrm{C}$ & 0.19 & $0.03 \mathrm{~B}$ & 0.08 & $0.03 \mathrm{~A}$ & 0.43 & $0.18 \mathrm{C}$ & 0.45 & $0.09 \mathrm{D}$ & 0.86 & $0.16 \mathrm{C}$ \\
\hline $22: 4 n-6$ & 0.63 & $0.15 \mathrm{D}$ & 2.11 & $0.25 \mathrm{E}$ & 2.04 & $0.61 \mathrm{~F}$ & 0.30 & $0.06 \mathrm{C}$ & 0.16 & $0.05 \mathrm{~A}$ & 0.22 & $0.09 \mathrm{~B}$ \\
\hline $22: 5 n-6$ & 0.35 & $0.06 \mathrm{~A}$ & 0.51 & $0.06 \mathrm{~B}$ & 0.46 & $0.03 \mathrm{~B}$ & 0.27 & $0.05 \mathrm{~A}$ & 0.28 & $0.05 \mathrm{~A}$ & 0.29 & $0.10 \mathrm{~A}$ \\
\hline $22: 5 n-3$ & 1.24 & $0.47 \mathrm{~B}$ & 3.58 & $0.44 \mathrm{C}$ & 5.40 & $0.36 \mathrm{D}$ & 0.86 & $0.18 \mathrm{~A}$ & 1.11 & $0.05 \mathrm{~B}$ & 1.32 & $0.47 \mathrm{~B}$ \\
\hline $22: 6 n-3$ & 16.71 & $1.71 \mathrm{C}$ & 17.44 & $3.28 \mathrm{C}$ & 6.72 & $0.81 \mathrm{~A}$ & 18.65 & $4.33 \mathrm{C}$ & 11.38 & $2.97 \mathrm{~B}$ & 9.12 & $1.94 \mathrm{AB}$ \\
\hline $24: 1 n-9$ & 0.43 & $0.12 \mathrm{~B}$ & 0.65 & $0.13 \mathrm{D}$ & 0.14 & $0.02 \mathrm{~A}$ & 0.59 & $0.16 \mathrm{CD}$ & 0.48 & $0.05 \mathrm{E}$ & 0.82 & $0.13 \mathrm{BC}$ \\
\hline
\end{tabular}

and straight- and branched-chain 17:0 compared to the pelagic fishes. The pelagic species contained more 14:0 than the demersal species. In monounsaturated fatty acids (MUFA), the demersal fishes were relatively rich in $17: 1 \mathrm{n}-8,18: 1 \mathrm{n}-13,18: 1 \mathrm{n}-7$ and the elongation product of the latter, i.e. 20:1n-7. One of the most striking differences in the pelagic and demersal fishes was that the pelagic species contained 5 to $10 \%$ (on molar basis) of 20:1n-9 and 22:1n-11, while these long-chain MUFA were only 1 to $3 \%$ in the demersal species. In polyunsaturated fatty acids (PUFA), the demersal fishes contained in general more of the long-chain PUFAs: $20: 4 n-6,20: 5 n-3,22: 4 n-6$ and 22:5n-3, but less of their 18-C precursors: 18:2n-6, 18:3n-3 and 18:4n-3.

\section{Transfer of fatty acids characteristic to the fish diets into herring gull plasma}

When the FAS of the dietary fishes and those of the plasma from gulls fed the corresponding fish for several weeks are compared, the first impression is that 
Table 1 (continued)

\begin{tabular}{|c|c|c|c|c|c|c|c|c|c|c|c|c|c|}
\hline \multirow[t]{3}{*}{ Fatty acid } & \multicolumn{12}{|c|}{ Herring gulls on specific fish diets $(\mathrm{N}=3-4)$} & \\
\hline & \multicolumn{2}{|c|}{ Haddock } & \multicolumn{2}{|c|}{ Whiting } & \multicolumn{2}{|c|}{ Flatfish } & \multicolumn{2}{|c|}{ Herring } & \multicolumn{2}{|c|}{$\begin{array}{l}\text { - Pelagic }- \\
\text { Sardine }\end{array}$} & \multicolumn{2}{|c|}{ Mackerel } & \\
\hline & Mean & $\mathrm{SD}$ & Mean & $\mathrm{SD}$ & Mean & $\mathrm{SD}$ & Mean & $\mathrm{SD}$ & Mean & $\mathrm{SD}$ & Mean & $\mathrm{SD}$ & \\
\hline 14:0 & 0.59 & $0.19 \mathrm{~A}$ & 0.67 & $0.06 \mathrm{~A}$ & 0.61 & $0.07 \mathrm{~A}$ & 2.08 & $0.40 \mathrm{C}$ & 1.42 & $0.39 \mathrm{~B}$ & 1.86 & $0.34 \mathrm{BC}$ & \\
\hline $15: 0$ iso & 0.09 & 0.05 & 0.29 & 0.17 & 0.23 & 0.09 & 0.18 & 0.18 & 0.16 & 0.19 & 0.25 & 0.15 & \\
\hline $15: 0$ & 0.35 & 0.18 & 0.30 & 0.05 & 0.35 & 0.02 & 0.36 & 0.02 & 0.51 & 0.06 & 0.48 & 0.05 & $\mathrm{p}<0.031$ \\
\hline $16: 0$ iso & 0.11 & 0.06 & 0.08 & 0.01 & 0.15 & 0.02 & 0.04 & 0.01 & 0.05 & 0.01 & 0.05 & 0.03 & $\mathrm{p}<0.033$ \\
\hline $16: 0$ & 22.40 & 1.51 & 22.74 & 1.11 & 23.92 & 0.87 & 24.19 & 0.51 & 23.26 & 1.80 & 22.21 & 0.75 & \\
\hline $16: 1 n-9$ & 0.20 & $0.01 \mathrm{~B}$ & 0.16 & $0.03 \mathrm{~B}$ & 0.27 & $0.01 \mathrm{C}$ & 0.26 & $0.02 \mathrm{C}$ & 0.12 & $0.03 \mathrm{~A}$ & 0.19 & 0.03 B & \\
\hline $16: 1 n-7$ & 1.60 & $0.35 \mathrm{AB}$ & 1.42 & $0.09 \mathrm{~A}$ & 2.08 & $0.12 \mathrm{AB}$ & 1.84 & $0.28 \mathrm{AB}$ & 2.37 & 0.64 B & 1.64 & $0.17 \mathrm{AB}$ & \\
\hline $16: 1 n-5$ & 0.10 & $0.04 \mathrm{AB}$ & 0.08 & $0.01 \mathrm{~A}$ & 0.08 & $0.01 \mathrm{~A}$ & 0.24 & $0.01 \mathrm{D}$ & 0.12 & $0.03 \mathrm{BC}$ & 0.15 & $0.01 \mathrm{C}$ & \\
\hline $16: 2 n-4$ & 0.01 & $0.01 \mathrm{~A}$ & 0.02 & $0.00 \mathrm{AB}$ & 0.05 & $0.01 \mathrm{BC}$ & 0.10 & $0.03 \mathrm{C}$ & 0.10 & $0.03 \mathrm{C}$ & 0.08 & $0.05 \mathrm{C}$ & \\
\hline $16: 3 n-4$ & 0.02 & 0.01 & 0.03 & 0.01 & 0.02 & 0.01 & 0.04 & 0.01 & 0.03 & 0.01 & 0.03 & 0.01 & \\
\hline $16: 4 n-1$ & 0.01 & $0.01 \mathrm{~A}$ & 0.02 & $0.01 \mathrm{AB}$ & 0.04 & $0.01 \mathrm{BC}$ & 0.08 & $0.02 \mathrm{D}$ & 0.05 & $0.02 \mathrm{C}$ & 0.03 & $0.01 \mathrm{AB}$ & \\
\hline 17:0 iso & 0.24 & 0.11 & 0.20 & 0.02 & 0.35 & 0.04 & 0.08 & 0.01 & 0.08 & 0.02 & 0.14 & 0.01 & $\mathrm{p}<0.006$ \\
\hline 17:0 anteiso & 0.18 & $0.08 \mathrm{~B}$ & 0.15 & $0.02 \mathrm{~B}$ & 0.36 & $0.04 \mathrm{C}$ & 0.05 & $0.01 \mathrm{~A}$ & 0.08 & $0.02 \mathrm{~A}$ & 0.09 & $0.01 \mathrm{~A}$ & \\
\hline $17: 0$ & 0.55 & 0.28 & 0.46 & 0.09 & 0.55 & 0.06 & 0.42 & 0.03 & 0.79 & 0.08 & 0.68 & 0.03 & $\mathrm{p}<0.023$ \\
\hline $17: 1 \mathrm{n}-8$ & 0.37 & 0.17 & 0.31 & 0.03 & 0.47 & 0.03 & 0.17 & 0.02 & 0.17 & 0.02 & 0.28 & 0.01 & $\mathrm{p}<0.016$ \\
\hline $18: 0$ & 17.59 & $0.63 \mathrm{~B}$ & 17.74 & $0.99 \mathrm{~B}$ & 17.57 & $0.28 \mathrm{~B}$ & 13.72 & $1.10 \mathrm{~A}$ & 17.09 & $0.87 \mathrm{~B}$ & 16.55 & 1.14 B & \\
\hline 18:1n-13 & 0.21 & 0.08 & 0.18 & 0.03 & 0.64 & 0.11 & 0.02 & 0.01 & 0.02 & 0.01 & 0.03 & 0.01 & $\mathrm{p}<0.005$ \\
\hline $18: 1 n-9+11$ & 19.70 & 7.17 & 19.61 & 2.28 & 18.03 & 1.57 & 14.47 & 1.20 & 11.30 & 0.55 & 16.10 & 0.63 & $\mathrm{p}<0.017$ \\
\hline $18: 1 n-7$ & 2.72 & $0.81 \mathrm{BC}$ & 2.28 & $0.19 \mathrm{BC}$ & 2.96 & $0.08 \mathrm{C}$ & 1.59 & $0.08 \mathrm{~A}$ & 1.90 & $0.23 \mathrm{AB}$ & 1.94 & $0.17 \mathrm{AB}$ & \\
\hline $18: 1 n-5$ & 0.23 & $0.08 \mathrm{BC}$ & 0.18 & $0.03 \mathrm{~B}$ & 0.15 & $0.01 \mathrm{~B}$ & 0.27 & $0.04 \mathrm{C}$ & 0.08 & $0.02 \mathrm{~A}$ & 0.22 & $0.02 \mathrm{BC}$ & \\
\hline $18: 2 n-6$ & 2.02 & $1.32 \mathrm{~A}$ & 2.23 & $0.58 \mathrm{~A}$ & 2.20 & $0.39 \mathrm{~A}$ & 5.71 & $1.00 \mathrm{AB}$ & 9.16 & $4.26 \mathrm{~B}$ & 2.99 & $1.73 \mathrm{~A}$ & \\
\hline $18: 2 n-4$ & 0.04 & $0.03 \mathrm{~A}$ & 0.04 & $0.01 \mathrm{AB}$ & 0.11 & $0.01 \mathrm{~B}$ & 0.06 & $0.02 \mathrm{AB}$ & 0.09 & $0.03 \mathrm{AB}$ & 0.06 & $0.01 \mathrm{AB}$ & \\
\hline $18: 3 n-3$ & 0.14 & $0.11 \mathrm{AB}$ & 0.19 & $0.05 \mathrm{~B}$ & 0.06 & $0.01 \mathrm{~A}$ & 0.58 & $0.06 \mathrm{C}$ & 0.34 & $0.06 \mathrm{C}$ & 0.42 & $0.10 \mathrm{C}$ & \\
\hline $18: 4 n-3$ & 0.08 & $0.04 \mathrm{~B}$ & 0.11 & $0.02 \mathrm{BC}$ & 0.02 & $0.01 \mathrm{~A}$ & 0.52 & $0.08 \mathrm{D}$ & 0.17 & $0.07 \mathrm{C}$ & 0.41 & $0.05 \mathrm{D}$ & \\
\hline 20:1n-11 & 0.22 & $0.10 \mathrm{AB}$ & 0.37 & $0.08 \mathrm{~B}$ & 0.17 & $0.03 \mathrm{~A}$ & 0.66 & $0.13 \mathrm{C}$ & 0.26 & $0.08 \mathrm{AB}$ & 0.67 & $0.09 \mathrm{C}$ & \\
\hline 20:1n-9 & 0.42 & $0.17 \mathrm{~A}$ & 0.84 & $0.18 \mathrm{~B}$ & 0.24 & $0.01 \mathrm{~A}$ & 2.71 & $0.59 \mathrm{C}$ & 0.72 & 0.13 B & 2.22 & $0.19 \mathrm{C}$ & \\
\hline $20: 1 n-7$ & 0.18 & 0.09 & 0.15 & 0.03 & 0.36 & 0.07 & 0.11 & 0.01 & 0.08 & 0.01 & 0.08 & 0.05 & $\mathrm{p}<0.018$ \\
\hline $20: 2 n-6$ & 0.21 & 0.10 & 0.17 & 0.02 & 0.14 & 0.02 & 0.18 & 0.01 & 0.15 & 0.02 & 0.14 & 0.02 & \\
\hline $20: 4 n-6$ & 14.57 & 3.48 B & 13.77 & $1.66 \mathrm{~B}$ & 14.48 & $0.47 \mathrm{~B}$ & 8.66 & $0.89 \mathrm{~A}$ & 10.66 & $0.61 \mathrm{AB}$ & 12.22 & $1.19 \mathrm{~B}$ & \\
\hline $20: 4 n-3$ & 0.15 & $0.09 \mathrm{AB}$ & 0.19 & $0.04 \mathrm{~B}$ & 0.04 & $0.01 \mathrm{~A}$ & 0.24 & $0.13 \mathrm{~B}$ & 0.14 & $0.09 \mathrm{AB}$ & 0.28 & 0.04 B & \\
\hline $20: 5 n-3$ & 4.85 & $0.31 \mathrm{~A}$ & 4.76 & $0.65 \mathrm{~A}$ & 5.47 & $0.78 \mathrm{AB}$ & 8.93 & $1.56 \mathrm{C}$ & 6.82 & 0.52 B & 5.70 & $0.89 \mathrm{AB}$ & \\
\hline $21: 5 n-3$ & 0.02 & $0.02 \mathrm{~A}$ & 0.05 & $0.02 \mathrm{AB}$ & 0.09 & $0.01 \mathrm{~B}$ & 0.06 & $0.03 \mathrm{AB}$ & 0.03 & $0.01 \mathrm{~A}$ & 0.04 & $0.01 \mathrm{~A}$ & \\
\hline $22: 1 n-11$ & 0.07 & $0.06 \mathrm{~A}$ & 0.32 & $0.13 \mathrm{~B}$ & 0.05 & $0.02 \mathrm{~A}$ & 0.98 & $0.21 \mathrm{C}$ & 0.30 & $0.09 \mathrm{~B}$ & 1.04 & $0.09 \mathrm{C}$ & \\
\hline $22: \ln -9$ & 0.01 & 0.01 & 0.04 & 0.01 & 0.02 & 0.01 & 0.06 & 0.01 & 0.01 & 0.00 & 0.06 & 0.01 & $\mathrm{p}<0.004$ \\
\hline $22: 4 n-6$ & 0.34 & $0.03 \mathrm{~B}$ & 0.31 & $0.07 \mathrm{~B}$ & 0.59 & $0.08 \mathrm{C}$ & 0.14 & $0.06 \mathrm{~A}$ & 0.22 & $0.05 \mathrm{AB}$ & 0.26 & $0.08 \mathrm{~B}$ & \\
\hline $22: 5 n-6$ & 0.29 & $0.02 \mathrm{~B}$ & 0.28 & $0.06 \mathrm{~B}$ & 0.29 & $0.04 \mathrm{~B}$ & 0.13 & $0.04 \mathrm{~A}$ & 0.51 & $0.15 \mathrm{C}$ & 0.32 & $0.05 \mathrm{~B}$ & \\
\hline $22: 5 n-3$ & 0.62 & $0.15 \mathrm{~A}$ & 0.66 & $0.12 \mathrm{~A}$ & 1.53 & $0.12 \mathrm{~B}$ & 0.40 & $0.03 \mathrm{~A}$ & 0.43 & $0.19 \mathrm{~A}$ & 0.50 & $0.06 \mathrm{~A}$ & \\
\hline $22: 6 n-3$ & 6.23 & 0.65 B & 6.36 & $0.90 \mathrm{~B}$ & 2.84 & $0.05 \mathrm{~A}$ & 7.32 & $0.23 \mathrm{~B}$ & 8.38 & $2.42 \mathrm{~B}$ & 7.15 & $0.54 \mathrm{~B}$ & \\
\hline $24: 1 n-9$ & 0.24 & $0.07 \mathrm{AB}$ & 0.31 & $0.03 \mathrm{AB}$ & 0.16 & $0.02 \mathrm{~A}$ & 0.35 & $0.05 \mathrm{AB}$ & 0.22 & $0.06 \mathrm{AB}$ & 0.37 & $0.01 \mathrm{~B}$ & \\
\hline
\end{tabular}

the FAS are very different (Table 1, Fig. 1). The FAS of gull plasma showed much higher proportions of 18:0 and 18:1n-9, and much less of the long-chain MUFA. In addition, in gull plasma, 20:4n-6 was greatly elevated at the expense of all the other PUFA when compared with the FAS of the dietary fish. Despite these differences of signature (due to the avian metabolism; see 'Discussion'), several characteristic features that can be linked to pelagic or demersal fish diets were found.

As a result of the dietary supply, in the plasma FAS of the gulls fed with pelagic fish the percentages of 14:0 were 2 to 3 times the values of the gulls fed with demersal fish, which on their part had higher per- centages of branched-chain (iso- and anteiso-) 17:0 (Table 1, Fig. 1). Secondly, the large supply of 20:1n-9 and 22:1n-11 from herring and mackerel produced relatively high levels of these acids in the gull plasma. However, the levels of these MUFA remained fairly low in the sardine-fed gulls, which had a feeding rate one-half that in the other dietary groups (see 'Discussion'). Thirdly, the proportions of 18C-PUFA: 18:2n-6, 18:3n-3 and 18:4n-3 were relatively high in the plasma of the gulls fed with pelagic fish, in accord with the birds' diet. Also in PCA biplots (objects and variables) the plasma samples of the gulls fed demersal and pelagic fish were clearly separated (Fig. 2). 

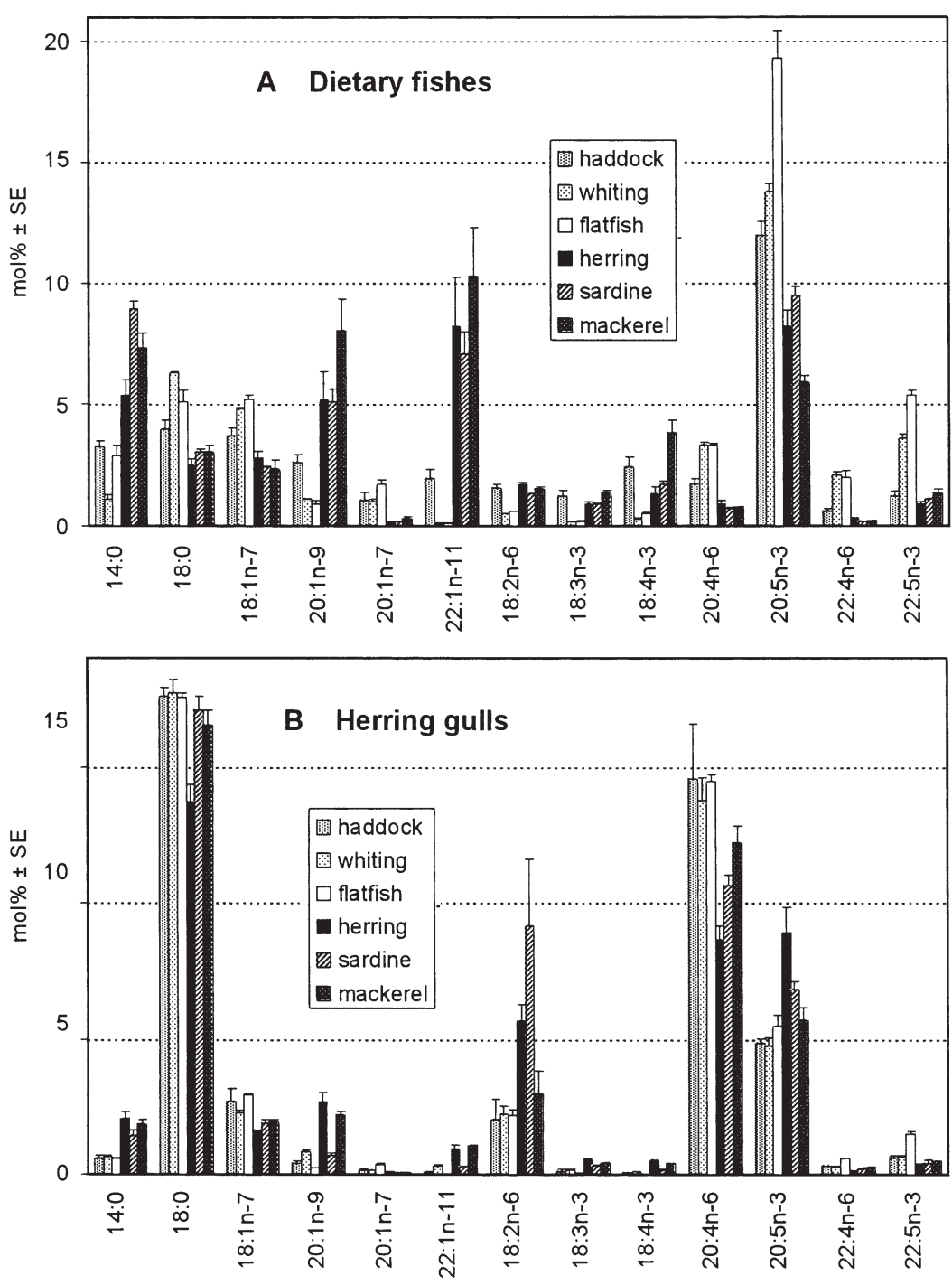

Fig. 1. Proportions of the major fatty acids (mol\%, mean $\pm \mathrm{SE}$ ) in demersal (haddock, whiting, flatfish) and pelagic fish (herring, sardine and mackerel; $N=6$ in each) that showed (A) the largest differences between demersal and pelagic fishes and (B) the proportions of these fatty acids in the herring gulls ( $\mathrm{N}=3$ to 4 ) fed these fishes

Specific fatty acid ratios that indicated whether the diet of the gulls consisted of demersal or pelagic fish were studied (Fig. 3). Since plasma lipid class composition (reflecting nutritional status) affects the plasma PUFA content and in field conditions partial oxidation of the sample PUFA may occur, these indicative ratios were calculated separately for PUFA and MUFA plus saturated fatty acids. The samples of demersal fish and plasma of herring gulls fed this fish had clearly larger values of the fatty acid ratios (iso-17:0 + anteiso-17:0 + $17: 1 \mathrm{n}-8) /(14: 0+20: 1 \mathrm{n}-9+22: 1 \mathrm{n}-11)$ and $(20: 4 \mathrm{n}-6) /$ $(18: 3 n-3+18: 4 n-3+20: 5 n-3)$ than the corresponding pelagic samples. Thus, these ratios may serve as an easily recognised indication of the importance of demersal fish in the diet of scavenging seabirds.

\section{Turnover rates of fatty acid signatures in herring gull plasma}

The FAS were determined every 5 or $7 \mathrm{~d}$ during the feeding tests. The switching of the diet affected the FAS of plasma total lipids already within this time in all test groups (Group A results shown in Fig. 4). Thus $5 \mathrm{~d}$ was enough to adopt a new component into the FAS or extinguish a considerable fatty acid component. In addition, the FAS of all the birds in each group responded to the changes of diet qualitatively and quantitatively in the same way. Only 1 of the 10 individuals used in this experiment responded exceptionally (see below).

In theory, the fatty mackerel and sardine should transfer a greater quantity of fatty acyl residues of lipids into the gull tissues than the other fishes (per fish weight). However, no marked differences in the rate of change of the FAS were found in the cases where the switch was from pelagic to demersal fish or vice versa (Fig. 4).

\section{Effects of nutritional state on fatty acid signatures in plasma}

To study the possible effects of transient changes in the nutritional status of the gulls on the FAS, both the lipid class and fatty acid compositions in the plasma of the gulls in Group B (haddock-herring) were followed. Although the plasma samples were taken $24 \mathrm{~h}$ after the last meal, the lipid contents of the birds' plasma varied considerably (Table 2). The main feature of the lipid profile that may affect the FAS of plasma total lipids are the proportions of fatty acids that originate from lipoprotein phospholipids ( 2 fatty acids per molecule) and neutral lipids, i.e. cholesterol esters ( 1 fatty acid per molecule) and triacylglycerols (3 fatty acids per molecule) (see 'Discussion'). It was calculated that in most of these gulls 17 to $31 \%$ of the plasma fatty acids originated from the neutral lipids. However, this variation in the lipid profile caused no such effects on FAS that would have 


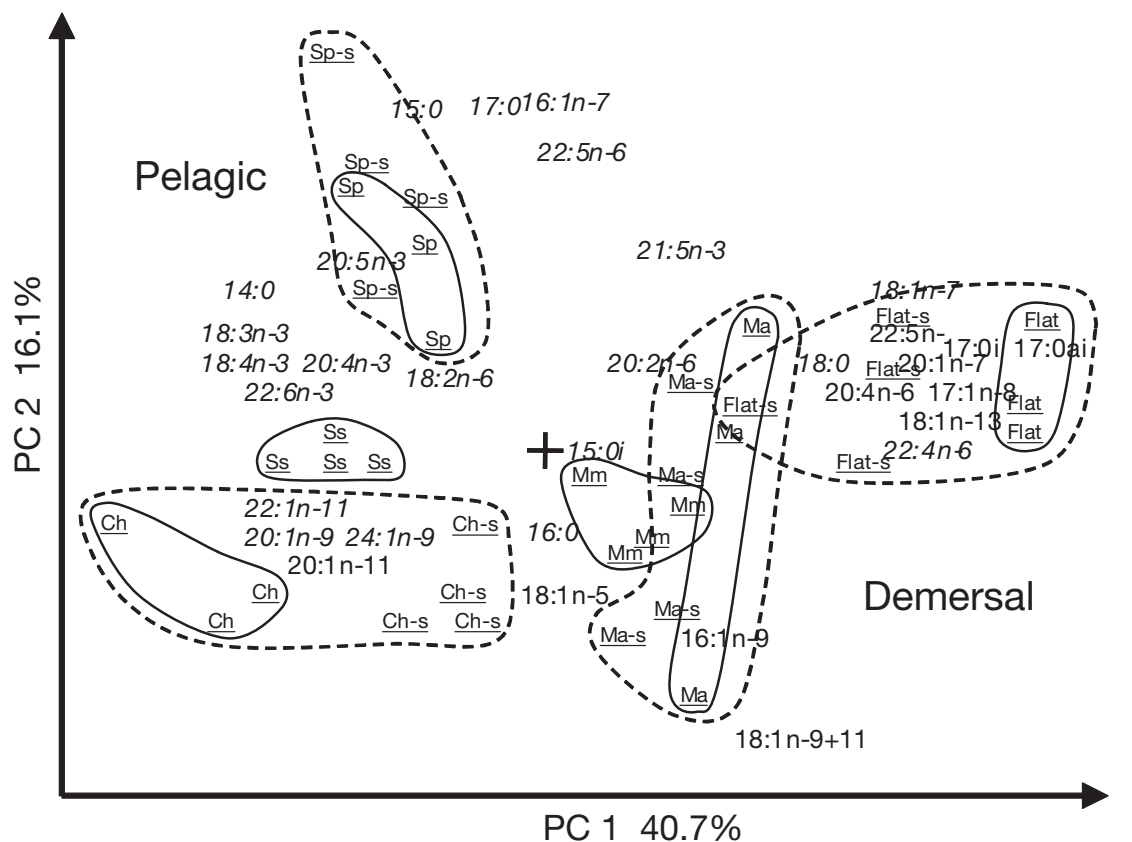

Fig. 2. PCA biplot of plasma fatty acids of the herring gulls fed the different fish diets. $\mathrm{Sp}=$ sardine, $\mathrm{Ss}=$ mackerel, $\mathrm{Ch}=$ herring, $\mathrm{Mm}=$ whiting, $\mathrm{Ma}=$ haddock, $\underline{\text { Flat }}=\overline{\text { flatfish sp. Additional }-\mathrm{s} \text { means } 2} \mathrm{wk}$ (instead of the $6 \mathrm{wk}$, no postfix) on the specific diet before the sampling

impaired their interpretation. In the previously mentioned individual that responded exceptionally to switching of diet, even $46 \%$ of plasma fatty acids originated from neutral lipids. This unusual plasma lipid profile raised the proportion of 18:1n-9, characteristic of neutral lipids, in the plasma of the bird, and

\section{A) Demersal SAT\&MUFA ratio in fish}

$(17: 0$ iso $+17: 0$ anteiso $+17: 1 n-8) /(14: 0+20: 1 n-9+22: 1 n-11)$

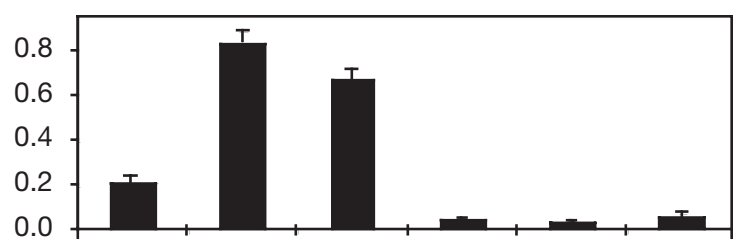

B) Demersal SAT\&MUFA ratio of gull plasma $(17: 0$ iso $+17: 0$ anteiso $+17: 1 n-8) /(14: 0+20: 1 n-9+22: 1 n-11)$

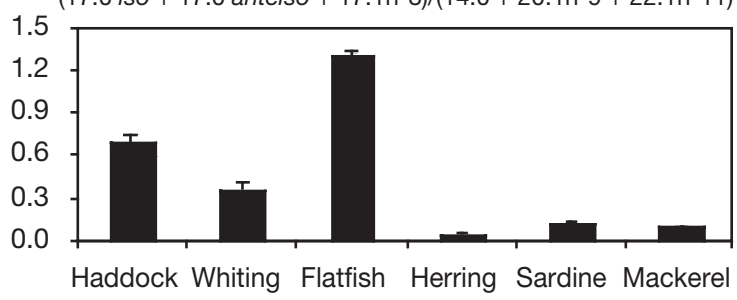

decreased the proportions of PUFA, abundant in phospholipids. However, even in this bird, the major markers of the diet, like the specific fatty acid ratios mentioned above indicated the diet accurately at every time of sampling.

\section{DISCUSSION}

The fatty acids of plasma total lipids originate mainly from phospholipids of the lipoprotein shelf and neutral lipids from the inner core of the lipoproteins. It is a general feature of vertebrate cells that fatty acyl residues that can be incorporated into the membrane phospholipids are more strictly and partly genetically controlled than the fatty acids of triacylglycerols and other neutral lipids that reflect dietary fatty acids more freely (Ackman \& Cunnane 1992). This was demonstrated recently for chick plasma lipids (García-Fuentes et al. 2002). It is also known that the plasma lipid profile varies according to the transient nutritional state of birds (Totzke et al. 1999). For example, in a few herring gull individuals of this study, the triacylglycerol concentrations of plasma were negligible, and did not provide enough material for lipid fractionation and subsequent fatty acid analysis. Although

C) Demersal PUFA ratio in fish

$$
(20: 4 n-6) /(18: 3 n-3+18: 4 n-3+20: 5 n-3)
$$

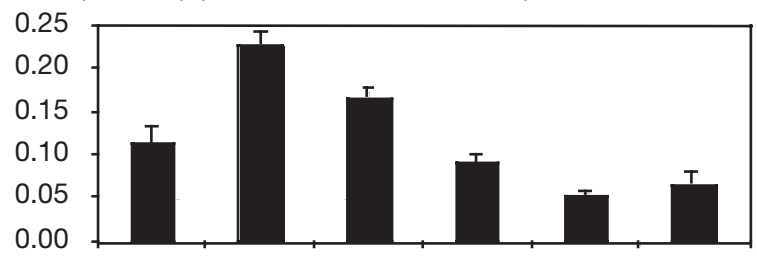

D) Demersal PUFA ratio of gull plasma $(20: 4 n-6) /(18: 3 n-3+18: 4 n-3+20: 5 n-3)$

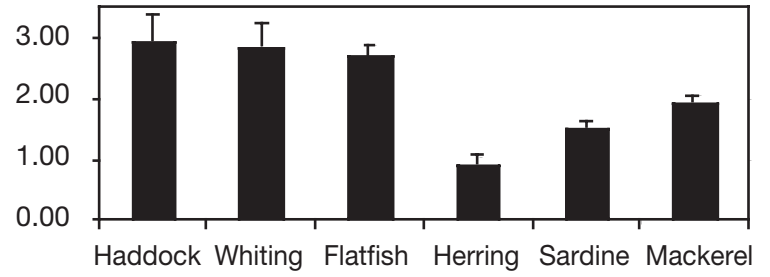

Fig. 3. Ratios (mol\%/mol\%) of saturated and monounsaturated fatty acids (SAT\&MUFA) (A,B), and polyunsaturated fatty acids (PUFA) $(C, D)$, in $(A, C)$ the dietary fish (mean $\pm S E, N=6$ ) and $(B, D)$ plasma of herring gulls (mean $\pm S E, N=3$ to 4$)$ fed the demersal (haddock, whiting and flatfish) and pelagic (herring, sardine and mackerel) fish 


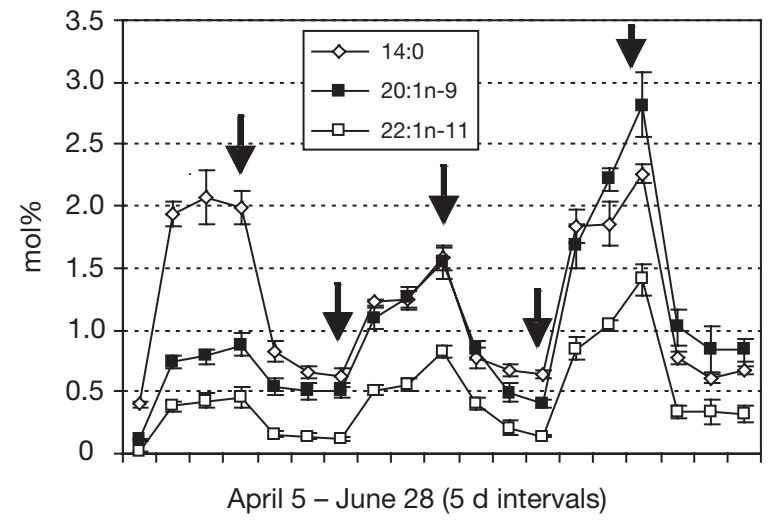

Fig. 4. Changes in the proportions (mol\%) of fatty acid markers 20:1n-9, 22:1n-11 and 14:0 in 4 herring gull individuals fed alternating diets of pelagic and demersal fish in the order: sardine, haddock, herring, flatfish mackerel and whiting. The switching of the diet is indicated as arrows. Plasma samples were studied for the fatty acids every $5 \mathrm{~d}$, and the values are means of 4 gulls $\pm \mathrm{SE}$

we did not try to assign the origin of different fatty acids to certain lipids, a crucial question for this calibration study is whether the lipid profiles of seabird plasma vary to such an extent that the accuracy of the dietary information obtained from the total lipid FAS is significantly impaired.

Our data showed that although the lipid profiles of the herring gull plasma varied (Table 2), the specific fatty acid ratios calculated indicated the diet accurately (Fig. 3). We have also determined plasma lipid class compositions in 72 great skuas from Shetland. In these wild birds, the proportions of fatty acids that originated from neutral lipids (16 to $49 \mathrm{~mol} \%$ ) were similar to the proportions found in the captive herring gulls of this study (17 to $46 \mathrm{~mol} \%$ ) (A. Käkelä et al. unpubl.). Thus, in terms of plasma lipid profiles, the herring gulls are similar to the wild scavenging species, and the results of this study very likely form valid reference data to be used for several related species of seabirds.

This calibration data, demonstrated either by multivariate approach (Fig. 2) or the indicative fatty acid ratios (Fig. 3), showed that the distinct differences of FAS between demersal and pelagic fish species caused clearly detectable differences in the FAS of seabird plasma. Due to the metabolic modifications in avian body, the dietary composition is never transferred as such, and the composition of plasma fatty acids may differ considerably from the original composition of the diet. However, several relative, even manifold, differ- ences were found in the percentages of certain fatty acids in plasma of the herring gulls fed demersal and pelagic fish.

The clearest markers of pelagic diet included the relatively high proportions of $18: 2 n-6,18: 3 n-3$, 18:4n-3 and 14:0 that are abundant in pelagic phytoplankton, and transferred in the food web further to fishes (Ackman et al. 1968, Ackman 1999, Napolitano 1999, Budge et al. 2002). The long-chain MUFA, 20:1n-9 and 22:1n-11, also transferred to the birds from the pelagic fish diet, originate from the alcohol moieties of zooplankton wax esters (Ratnayake \& Ackman 1979, Saito \& Kotani 2000, Dalsgaard et al. 2003). In addition to having low levels of the pelagic markers, the birds fed demersal fish also have a few features characteristic of benthic food webs in their FAS. Microbial lipids of North Sea sediments are rich in odd- and branched-chain saturated fatty acids, and the isomer distribution of their MUFA is different from that of the pelagic fatty acid sources and favours for example 18:1n-7 (Stoeck et al. 2002). Consequently, the plasma FAS of the birds fed demersal fish contained relatively high percentages of iso-17:0, anteiso-17:0, 17:0 and 18:1n-7.

Although there was much more 20:5n-3 than 20:4n-6 available in both demersal and pelagic fish, 20:4n-6 clearly dominated in bird plasma. This is most likely due to the evolutionary biochemical preferences to incorporate only certain fatty acids into sn-1 and sn-2 positions of lipoprotein phospholipids (Brockerhoff \& Ackman 1967, Brockerhoff et al. 1967). Since birds evolved from terrestrial reptiles, which obtained more n-6 PUFA than n-3 PUFA from their diet, it is likely that they retained a preference to incorporate 20:4n-6 into their structural lipids. Consequently, a slightly larger supply of 20:4n-6 from demersal fish caused clearly higher ratios of 20:4n-6 to 20:5n-3 and C18-PUFA in plasma of the birds fed the demersal diets (Figs. 1 \& 3). Fishes that feed on detritus or are top predators are both normally rich in 20:4n-6 (e.g. Kuusipalo \& Käkelä 2000, Budge et al. 2002).

Table 2. Concentrations of major lipid classes in the plasma of herring gulls fed haddock followed by herring (Group B, N = 3 sampled weekly during 5 or 6 wk). The variations due to sampling time were larger than the variations between the individual gulls

\begin{tabular}{|c|c|c|}
\hline & $\begin{array}{l}\text { Haddock-fed gulls } \\
(\mathrm{N}=3 / \mathrm{wk} \times 5 \mathrm{wk}) \\
\text { Mean } \pm \mathrm{SD} \text { (range) }\end{array}$ & $\begin{array}{l}\text { Herring-fed gulls } \\
(\mathrm{N}=3 / \mathrm{wk} \times 6 \mathrm{wk}) \\
\text { Mean } \pm \mathrm{SD} \text { (range) }\end{array}$ \\
\hline Phospholipids $\left(\mathrm{g} \mathrm{l}^{-1}\right)$ & $7.8 \pm 1.4(5.1-10.5)$ & $9.1 \pm 2.6(5.0-14.9)$ \\
\hline Cholesteryl esters $\left(\mathrm{g} \mathrm{l}^{-1}\right)$ & $4.3 \pm 1.2(2.2-6.6)$ & $4.7 \pm 2.0(1.5-8.2)$ \\
\hline Cholesterol $\left(\mathrm{g} \mathrm{l}^{-1}\right)$ & $1.1 \pm 0.3(0.6-1.8)$ & $1.2 \pm 0.5(0.4-2.1)$ \\
\hline Triacylglycerols $\left(\mathrm{g} \mathrm{l}^{-1}\right.$ ) & $0.11 \pm 0.05(0.04-0.21)$ & $0.66 \pm 1.59(0.005-6.81)$ \\
\hline Fatty acids $\left(\mathrm{mg} \mathrm{l}^{-1}\right)$ & $36 \pm 32(4-159)$ & $22 \pm 27(1-107)$ \\
\hline
\end{tabular}


Less than $5 \mathrm{~d}$ feeding on certain prey item was enough to produce recognisable marks in the plasma FAS of the herring gulls. Thus, the signatures can be used to assess the recent diet of the seabirds. In addition, it is expected that birds eating a mixed diet would show intermediate FAS between the extreme values from solely demersal or pelagic fish diet. However, when estimating the proportions of each type of prey, additional factors, e.g. the lipid contents of the fish, absorption efficiencies of different lipids and even bird age have to be considered (Ward \& Marquardt 1983). Also, during negative energy balance birds may mobilise the fatty acid resources of their liver and adipose tissues, and these fatty acids representing earlier meals are subsequently transported in plasma to be oxidized and esterified in other tissues and recycled back to circulation. In herring gulls, plasma concentrations of cholesterol and triacylglycerol were found to increase during experimental fasting (Totzke et al. 1999). Thus, the plasma FAS may also show some signs of long-term dietary background. For example, the herring gulls in Group A (as all the other birds before the experiment) were fed basal pellet feed that contained large amounts of saturated fatty acids, MUFA and 18:2n-6 as the major PUFA. When the gulls were offered sardine, the consumption rate was only onehalf that when the birds were fed the other fish diets. Consequently, in the plasma FAS of these birds, the pelagic markers showed unexpectedly low proportions, except the 18:2n-6, apparently released from long-term stores, which showed unexpectedly high proportions.

The results of this calibration study suggest that FAS of plasma total lipids can be used to estimate the proportions of discarded demersal fish and pelagic fish, which have very different FAS, in the diet of scavenging seabirds. As demonstrated, the use of plasma FAS benefits from the fact that transient changes in nutritional status do not significantly impair the accuracy of this approach. Plasma samples are ideal to study the recent diet of the birds. For assessing the long-term dietary background, however, fatty acids of carefully selected adipose tissues that have a slow turnover rate of fatty acids must be studied.

Acknowledgements. This study was supported by EU contract Q5RS-2001-00839 'DISCBIRD'.

\section{LITERATURE CITED}

Ackman RG (1992) Application of gas-liquid chromatography to lipid separation and analysis: qualitative and quantitative analysis. In: Chow CK (ed) Fatty acids in foods and their health implications. Marcel Dekker, New York, p 47-63
Ackman RG (1999) Comparison of lipids in marine and freshwater organisms. In: Arts MT, Wainman BC (eds) Lipids in freshwater ecosystems. Springer-Verlag, New York, p 263-298

Ackman RG, Cunnane C (1992) Long-chain polyunsaturated fatty acids: sources, biochemistry and nutritional/clinical applications. Adv Appl Lipid Res 1:161-215

Ackman RG, Tocher CS, McLachlan J (1968) Marine phytoplankter fatty acids. J Fish Res Board Can 25:1603-1620

Alverson DL, Freeberg MH, Murawski SA, Pope JG (1994) A global assessment of fisheries by catch and discards. FAO Fisheries Technical Paper 339. FAO, Rome

Bearhop S, Waldron S, Votier SC, Furness RW (2002) Factors that influence assimilation rates and fractionation of nitrogen and carbon stable isotopes in avian blood and feathers. Physiol Biochem Zool 75:451-458

Bradshaw CJ, Hindell MA, Best NJ, Phillips KL, Wilson G, Nichols PD (2003) You are what you eat: describing the foraging ecology of southern elephant seals (Mirounga leonina) using blubber fatty acids. Proc R Soc Lond B 270: 1283-1292

Brockerhoff H, Ackman RG (1967) Positional distribution of isomers of monoenoic fatty acids in animal glycerolipids. J Lipid Res 8:661-666

Brockerhoff H, Hoyle RJ, Hwang PC (1967) Incorporation of fatty acids of marine origin into triglycerides and phospholipids of mammals. Biochim Biophys Acta 144:541-548

Budge SM, Iverson SJ, Bowen WD, Ackman RG (2002) Among-and within-species variability in fatty acid signatures of marine fish and invertebrates on the Scotian Shelf, Georges Bank, and southern Gulf of St. Lawrence. Can J Fish Aquat Sci 59:86-898

Christie WW (1993) Preparation of ester derivatives of fatty acids for chromatographic analysis. In: Christie WW (ed) Advances in lipid methodology - II. Oily Press, Dundee, p 69-111

Dahl TM, Falk-Petersen S, Gabrielsen GW, Sargent JR, Hop H, Millar RM (2003) Lipids and stable isotopes in common eider, black-legged kittiwake and northern fulmar: a trophic study from an Arctic fjord. Mar Ecol Prog Ser 256: 257-269

Dalsgaard J, St. John M, Kattner G, Müller-Navarra D, Hagen W (2003) Fatty acid trophic markers in the pelagic marine environment. Adv Mar Biol 46:225-340

FAO (1995) Code of conduct for responsible fisheries. FAO, Rome

Folch J, Lees M, Sloane-Stanley GH (1957) A simple method for the isolation and purification of total lipides from animal tissues. J Biol Chem 226:497-509

Frank D (1992) The influence of feeding conditions on food provisioning of chicks in common terns Sterna hirundo nesting in the German Wadden Sea. Ardea 80:45-55

Furness RW (2003) Impacts of fisheries on seabird communities. Sci Mar 67(Suppl 2):33-45

García-Fuentes E, Gil-Villarino A, Zafra MF, García-Peregrín (2002) Differential changes in the fatty acid composition of the main lipid classes of chick plasma induced by dietary coconut oil. Comp Biochem Physiol B 133:269-275

Garthe S, Camphuysen CJ, Furness RW (1996) Amounts of discards by commercial fisheries and their significance as food for seabirds in the North Sea. Mar Ecol Prog Ser 136: $1-11$

Grahl-Nielsen O, Andersen M, Derocher AE, Lydersen C, Wiig Ø, Kovacs KM (2003) Fatty acid composition of the adipose tissue of polar bears and of their prey: ringed seals, bearded seals and harp seals. Mar Ecol Prog Ser 265:275-282 
Hilderbrand GV, Farley SD, Robbins CT, Hanley TA, Titus K, Servheen C (1996) Use of stable isotopes to determine diets of living and extinct bears. Can J Zool 74: 2080-2088

Hobson KA, Piatt JF, Pitocchelli J (1994) Using stable isotopes to determine seabird trophic relationships. J Anim Ecol 63: 786-798

Hüppop O, Wurm S (2000) Effects of winter fishery activities on resting numbers, food and body condition of large gulls Larus argentatus and L. marinus in the south-eastern North Sea. Mar Ecol Prog Ser 194:241-247

ICES (2002) Report of the ICES Advisory Committee on Fishery Management, 2002. Cooperative Research Report No. 255. ICES, Copenhagen

Iverson SJ, Field C, Bowen WD, Blanchard W (2004) Quantitative fatty acid signature analysis: a new method of estimating predator diets. Ecol Monogr 74:211-235

Iverson SJ, Frost KJ, Lowry LF (1997) Fatty acid signatures reveal fine-scale structure of foraging distribution of harbour seals and their prey in Prince William Sound, Alaska. Mar Ecol Prog Ser 151:255-271

Käkelä R, Hyvärinen H (1996) Site-specific fatty acid composition in adipose tissues of several northern aquatic and terrestrial mammals. Comp Biochem Physiol B 115: 501-514

Käkelä R, Pölönen I, Miettinen M, Asikainen J (2001) Effects of different fat supplements on growth and liver lipids and fatty acids of mink. Acta Agric Scand A 51:217-223

Kuusipalo L, Käkelä R (2000) Muscle fatty acids as indicators of niche and habitat in Malawian cichlids. Limnol Oceanogr 45:996-1000

Kvalheim OM, Karstang TV (1987) A general-purpose program for multivariate data analysis. Chemom Intell Lab Syst 2:235-237

Mitchell PI, Newton SF, Ratcliffe N, Dunn TE (2004) Seabird populations of Britain and Ireland. T \& AD Poyser, London

Napolitano GE (1999) Fatty acids as trophic and chemical markers in freshwater ecosystems. In: Arts MT, Wainman BC (eds) Lipids in freshwater ecosystems. SpringerVerlag, New York, p 21-44

Olsen E, Grahl-Nielsen O (2003) Blubber fatty acids of minke whales: stratification, population identification and relation to diet. Mar Biol 142:13-24

Oro D, Furness RW (2002) Influences of food availability and predation on survival of kittiwakes. Ecology 83:2516-2528

Oro D, Pradel R (2000) Determinants of local recruitment in a growing colony of Audouin's gull. J Anim Ecol 69:1-14

Oro D, Ruiz X (1997) Exploitation of trawler discards by breeding seabirds in the north-west Mediterranean: differences between the Ebro Delta and the Balearic Islands areas. ICES J Mar Sci 54:695-707

Oro D, Aguilar JS, Igual JM, Louzao M (2003) Modeling

Editorial responsibility: Otto Kinne (Editor-in-Chief), Oldendorf/Luhe, Germany demography and extinction risk in the endangered Balearic shearwater. Biol Conserv 116:93-102

Ratcliffe N, Furness RW, Hamer KC (1998) The interactive effects of age and food supply on the breeding ecology of great skuas. J Anim Ecol 67:853-862

Ratcliffe N, Catry P, Hamer KC, Klomp NI, Furness RW (2002) The effect of age and year on the survival of breeding adult great skuas Catharacta skua in Shetland. Ibis 144: 384-392

Ratnayake WN, Ackman RG (1979) Fatty alcohols in capelin, herring and mackerel oils and muscle lipids. II. A comparison of fatty acids from wax esters with those of triglycerides. Lipids 14:804-810

Rau GH, Ainley DG, Bengtson JL, Torres JJ, Hopkins TL (1992) 15N/14N and 13C/12C in Weddell Sea birds, seals, and fish: implications for diet and trophic structure. Mar Ecol Prog Ser 84:1-8

Rouvinen K, Kiiskinen T (1989) Influence of dietary fat source on the body fat composition of mink (Mustela vison) and blue fox (Alopex lagopus). Acta Agric Scand 39:279-288

Saito H, Kotani Y (2000) Lipids of four boreal species of calanoid copepods: origin of monoene fats of marine animals at higher trophic levels in the grazing food chain in the subarctic ocean ecosystem. Mar Chem 71:69-82

Soriguer FJ, Tinahones FJ, Monzón A, Pareja A, RojoMartínez G, Moreno F, Esteva I, Gómez-Zumaquero JM (2000) Varying incorporation of fatty acids into phospholipids from muscle, adipose and pancreatic exocrine tissues and thymocytes in adult rats fed with diets rich in different fatty acids. Eur J Epidemiol 16:585-594

Stoeck T, Kröncke I, Duineveld GCA, Palojärvi A (2002) Phospholipid fatty acid profiles at depositional and non-depositional sites in the North Sea. Mar Ecol Prog Ser 241:57-70

Stratoudakis Y, Fryer RJ, Cook RM (1998) Discarding practices for commercial gadoids in the North Sea. Can J Fish Aquat Sci 55:1632-1644

Totzke U, Fenske M, Hüppop O, Raabe H, Schach N (1999) The influence of fasting on blood and plasma composition of herring gulls (Larus argentatus). Physiol Biochem Zool 72:426-437

Votier SC, Bearhop S, Ratcliffe N, Furness RW (2001) Pellets as indicators of diet in great skuas Catharacta skua. Bird Study 48:373-376

Votier SC, Bearhop S, MacCormick A, Ratcliffe N, Furness R W (2003) Assessing the diet of great skuas, Catharacta skua, using five different techniques. Polar Biol 26:20-26

Votier SC, Furness RW, Bearhop S, Crane JE and 11 others (2004) Changes in fisheries' discard rates and seabird communities. Nature 427:727-730

Ward AT, Marquardt RR (1983) The effect of saturation, chain length of pure triglycerides, and age of bird on the utilization of rye diets. Poult Sci 62:1054-1062

Submitted: June 24, 2004; Accepted: December 28, 2004

Proofs received from author(s): May 5, 2005 